\title{
Fracture behavior of shear key structures with a softening process zone
}

\author{
YOSHIO KANEKO ${ }^{1}$ and VICTOR C. $\mathrm{LI}^{2}$ \\ ${ }^{1}$ Design Division, Shimizu Corporation, Tokyo, Japan \\ ${ }^{2}$ Department of Civil Engineering, University of Michigan, Ann Arbor, Michigan 48109-2125 USA
}

Received 25 July 1991; accepted in revised form 24 August 1992

\begin{abstract}
The objective of this paper is to investigate the fracture behavior of short fiber reinforced ceramic structures by means of the fracture mechanics approach. In this paper, structural stability in relation to crack growth in shear key structures with a softening process zone under effectively bending loads is studied through residual strength diagrams and load deflection curves. In addition, the behavior of process zone size preceding traction free crack in the shear key structure is investigated.

Shear key structures with a softening process zone can behave stably under loading in the presence of a crack. Results of this analytical study potentially indicate that short fiber reinforcement for ceramics could eliminate catastrophic failure or unstable fracture behavior of ceramic structures, and also indicate the plausible applicability of ceramics as construction materials.
\end{abstract}

\section{Nomenclature}

The following symbols are used in this paper:

\begin{tabular}{|c|c|c|c|}
\hline$\sigma_{p}$ & $=$ applied load; & $E$ & $=$ Young's modulus; \\
\hline$\Delta$ & $\begin{array}{l}=\text { deflection at the center point of } \\
\text { loaded area; }\end{array}$ & $\delta_{c} \quad f^{*} \delta$ & $=$ critical CTOD \\
\hline$a_{0}$ & $\begin{array}{l}=\text { initial crack length (traction free } \\
\text { crack length) }\end{array}$ & $G_{f}=\frac{f_{i} o_{c}}{2}$ & $\begin{array}{l}=\text { fracture energy (for linear stress- } \\
\text { separation curve); }\end{array}$ \\
\hline $\begin{array}{l}l_{p} \\
\left(l_{p}\right)_{s s} \\
L\end{array}$ & $\begin{array}{l}=\text { process zone size; } \\
=\text { steady-state process zone size; } \\
=\text { size of a structure; }\end{array}$ & $l_{c h}=\frac{E G_{f}}{f_{t}^{2}}$ & $=$ material characteristic length; and \\
\hline $\begin{array}{l}f_{t} \\
f_{t}^{*} \\
v(=0.2)\end{array}$ & $\begin{array}{l}=\text { first cracking strength of composites; } \\
=\text { post cracking strength of composites; } \\
=\text { Poisson's ratio; }\end{array}$ & $K_{\mathrm{IC}}=\sqrt{ }$ & $=$ fracture toughness (for LEFM \\
\hline
\end{tabular}

\section{Introduction}

Previously, a qualitative analysis on applicability of advanced ceramics to construction based on a set of criteria such as durability, brittleness or economy was carried out [1], and some feasible structural systems for ceramics such as segmental precast structures were investigated [2]. Most of the introduced structural systems have a shear key. A shear key could be loaded critically in precast members since that part may have minimum area, or may have the largest stress. The fracture behavior of the shear key could thus control the behavior of the whole structure. It is the purpose of this paper to investigate the fracture behavior of the shear key structure made of short fiber reinforced ceramics by studying a mode I-dominated fracture of shear keys with a softening process zone. 


\section{Review of behavior of fiber reinforced composites}

The fracture behavior of fiber reinforced cementitious composite was studied by Li and Liang [3]. They analyzed the overall load-deformation behavior of the center-cracked panel shown in Fig. 1 in terms of load-crack opening displacement (COD) curves with the same fracture energy but different shapes. The stress-separation constitutive models used (see Fig. 2) are

(a) a linear straight line descending from the tensile strength at zero material separation to zero stress at the critical separation and

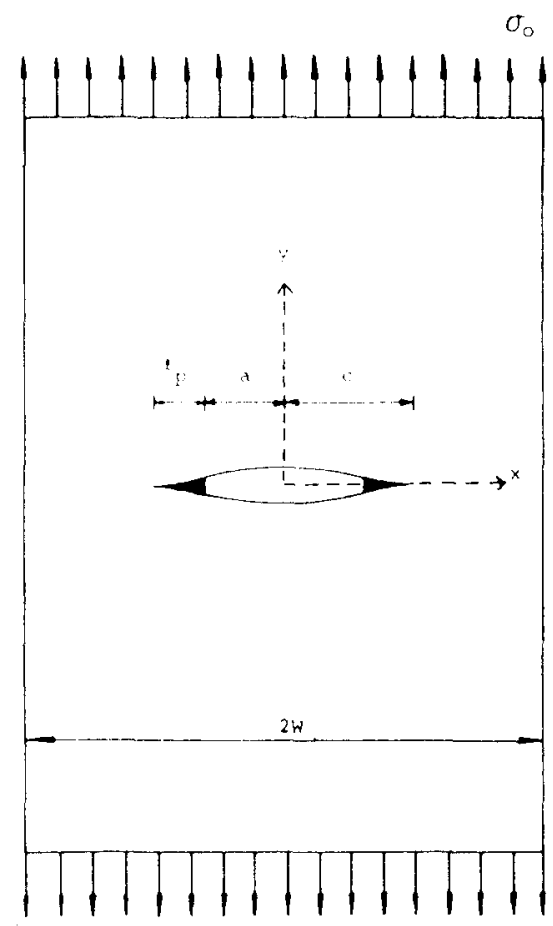

Fig. 1. Geometry of center-cracked panel; Traction Free Crack (TRF) with length $a$ and process zone with length $l_{p}$ make up total crack of length $c$ [3].

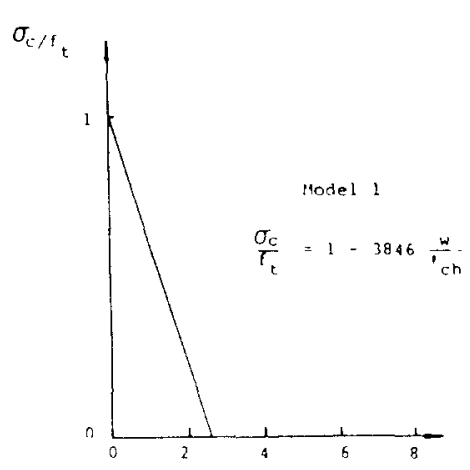

(a)

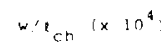

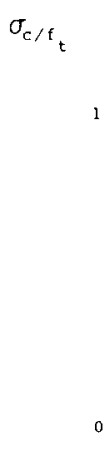

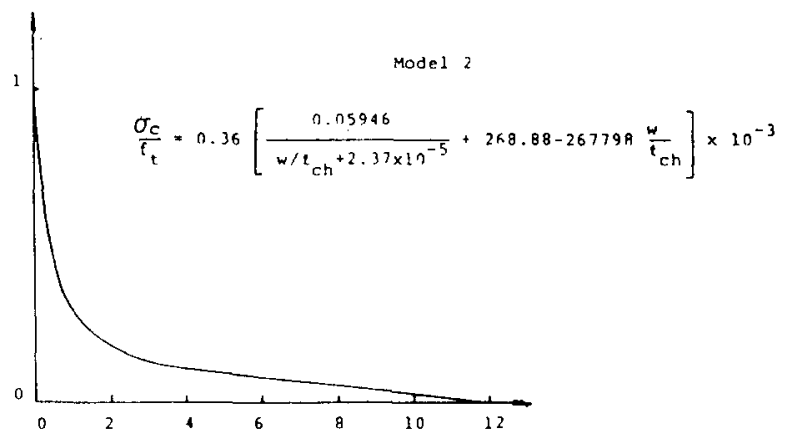

(b)

$\left(w / x_{c h} \times 10^{4}\right)$

Fig. 2. Stress-separation constitutive models; both models have same $G_{\mathrm{C}}$ and $f_{\mathrm{t}}$ but shapes are quite different with very small critical separation distance $w_{C}$ for (a) Model 1, and much larger $w_{C}$ for (b) Model 2, [3]. 


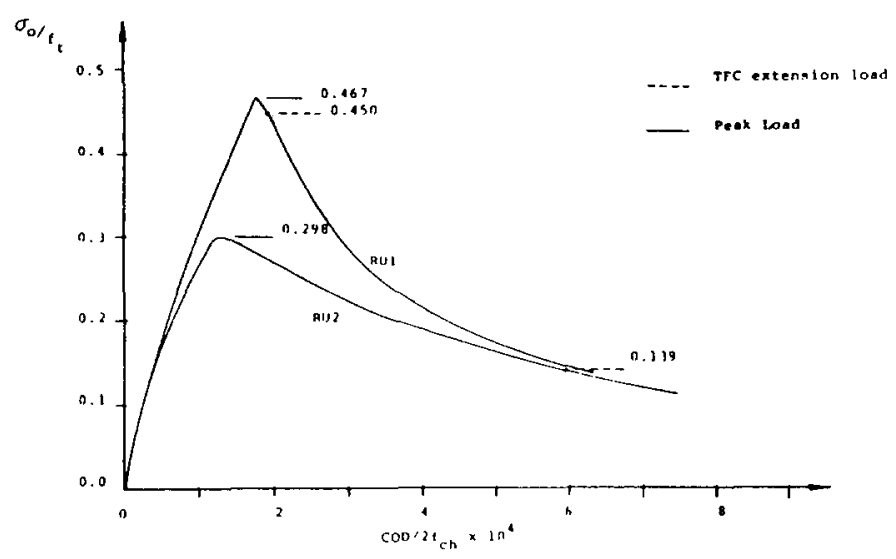

Fig. 3. Normalized load applied at remote edges of the center-cracked panel versus normalized opening displacement at crack center, for Model 1 (RU1) and for Model 2 (RU2) [3].

(b) a nonlinear line with a rapid drop in traction transfer with separation, followed by a long tail.

Their results (see Fig. 3) show the linear model allows the structure to reach a higher peak load, about one and a half times that of the nonlinear model. At the same time, the descending branch is much sharper in the load-deformation curve for the linear line model than for the nonlinear line model. These behaviors make the structure seemingly stronger (with higher tensile strength) but more brittle for the linear line model. Also their results show the process zone is still being developed at the peak load and after the peak load the traction free crack propagates when the process zone is fully developed. The tensile strength in the curve of fiber reinforced ceramics can be much higher than that of fiber reinforced concrete. As a result, the descending branch in the stress-separation curve of fiber reinforced ceramics might be sharper than that of fiber reinforced concrete. (Details of the stress-separation curve and the presence of multiple-cracking are controlled by fiber volume fraction, amongst other factors. In general, a higher fiber volume is used in ceramics than in concrete composites). From this limited point of view, the behavior of fiber reinforced ceramic structures may be predicted by a linear line model in the stressseparation curve while that of fiber reinforced concrete structures should generally be predicted by a nonlinear line model. In addition, we could calculate from their work the ratio of the traction free crack propagation load to the peak load, and the ratio of the COD at traction free crack propagation to that at the peak load. The former ratio for the linear line model is 0.95 and that for the nonlinear line model is 0.45 , and the latter ratio for the linear line model is 1.1 and that for the nonlinear line model is 5.0. For a stress-separation curve with a sharply descending softening branch (such as in the linear model described above), traction free crack propagation load can be assumed to be the same as the peak load. In this paper, this assumption is used for our analysis.

\section{Objective of analysis}

The present numerical study focuses on the investigation of fracture behavior in terms of residual strength diagram and load-deflection curve after the peak load (the pre-peak behavior is not considered) for different shapes of stress-separation curves. These stress-separation curves are determined by several types of assumed uniaxial tensile behaviors. Unlike conventional 
construction structures such as steel structures or steel reinforced concrete structures, ceramic structures may be adversely influenced by their unstable behavior after the peak load (or after propagation of the traction free crack). Therefore, we focus especially on the stability in relation to crack growth in the structures. The structural stability of ceramic structures is analyzed via the descending branch of both residual strength diagrams and load-deflection curves.

The behavior of fiber reinforced ceramic structures could be dominated by the process zone undergoing inelastic deformation described by a stress-separation curve. Li and Liang [3] suggested that process zone size $l_{p}$ depends on the stress-separation constitutive behavior, the loading configuration and the structural geometry. In the present analysis, using a similar approach to that of Li and Liang, we try to find the quantitative relation between process zone size $l_{p}$, the stressseparation constitutive behavior, and structural geometry (size) for a fixed loading configuration.

The above mentioned analysis can be carried out by means of a nonlinear elastic fracture mechanics (NLEFM) approach since they behave nonlinearly with the developed process zone. On the other hand, a linear elastic fracture mechanics (LEFM) approach is generally much less difficult than the NLEFM approach. Also, there are currently many general purpose computer programs in terms of LEFM concept rather than those in terms of NLEFM concept. Therefore, it is very important to find the valid condition for LEFM in the numerical process from the present simple model. This is also one of the objectives in this numerical analysis. Therefore, the behavior of shear key structures is also analysed by means of a LEFM approach, and compared with NLEFM results to determine the condition of validity for the LEFM approach.

\section{Numerical implementation}

Most short fiber reinforced ceramics are tension-softening materials which have decreasing post peak tensile strength with increasing displacement. During tensile testing of this material, specimens do not exhibit signs of plasticity prior to failure as metals do. Instead, a highly localized zone of straining eventually forms a through crack before final failure of the specimen. It has been observed that displacements along the post peak stress-strain curve are mainly due to the opening of the locally strained region which can also be described as a crack with incompletely separated surfaces across which stresses can still be transmitted. These observations have led to the concept of the tension-softening or stress-separation curve which relates post peak stresses across the crack to the crack opening displacement $\delta$. For fracture failure of a short fiber reinforced ceramic structure, it is assumed that a fictitious crack (a crack with bridging stresses) forms when the maximum strength of the material is reached. The bridging stresses decrease with increasing opening displacement until the separation reaches a critical value $\delta_{c}$ at which point the crack faces are separated completely and the bridging stresses cease to exist.

The effective crack may be considered as consisting of two parts:

(1) the real or traction free crack and

(2) the process zone with bridging stresses depending on the opening displacement along the process zone as prescribed by the material stress-separation curve.

Figure 4 shows a schematic diagram of this so called 'cohesive zone model'. In contrast to the singular stress distribution obtained from LEFM assumptions, stresses from the cohesive zone model do not exceed the material strength. Furthermore, propagation of the traction free crack is governed by the opening displacement $\delta^{*}$ at the crack tip of the traction free crack region. 
(a)

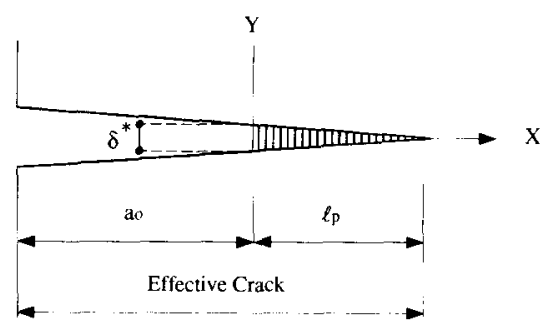

(b)

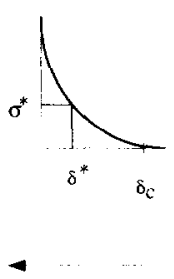

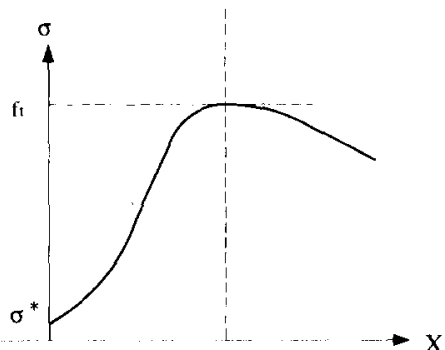

Fig. 4. The cohesive zone model: (a) Effective crack and (b) Stress distribution along the effective crack.

When $\delta^{*}=\delta_{c}$, free crack extension is imminent. This condition may be regarded as a replacement of the $K_{\mathrm{IC}}$-fracture criterion.

The cohesive zone model is actually based on Barenblatt's theory of equilibrium cracks [4]. According to the theory, the complete response of a cracked body to loads is defined by the stresses, the strains and the final configuration of the cracks which would make the crack tip stresses finite. Thus, unlike the typical elasticity problem, the final boundaries of the body or specifically the crack lengths are unknown. To find the complete solution, the molecular forces of cohesion which are activated along the separating faces of the crack must be included in the analysis. Although Barenblatt had considered inter-molecular stress-separation curves in his theory, his mathematical formulation of the problem is equally applicable to the cohesive zone model of tension-softening materials with their macroscopic stress-separation curves. The inclusion of the process zone size as another unknown is balanced by the requirement of zero singularities at the crack tips, i.e. the stress intensity factors induced by external loading must be cancelled out by the negative stress intensity factors induced by the cohesive stresses along the process zone. With this last requirement, the process zone size can be calculated.

Based on the above mentioned approach for tension softening materials, the numerical simulation was carried out by means of the hybrid boundary element method [5] and [6].

\section{Models for analysis}

The geometric and loading configurations of the analytical shear key model are shown in Fig. 5. The external load tends to open the corner which has a pre-existing crack $a_{0}$. For this structural geometry and loading configuration, only mode I crack may result.

The composite uniaxial curves used are shown in Figs. 6-8. These curves could express three typical types of stress-strain curves under tensile loading in short fiber reinforced ceramics. As discussed in the previous section, the stress-separation curves are assumed linear lines from three typical types of stress-strain curves.

These curves show the behavior of composites with different fiber reinforcement such as the characteristics of fibers, the distribution and the interfacial behaviors. In general, the interface is characterized by a frictional bond and a chemical bond (see Fig. 9). If only a frictional bond is present, there is stable transition from fiber debonding to fiber pull-out. If there is a chemical bond as well, stable debonding will be followed by an unstable drop to the pull-out branch [7].

In the softening model 1 , extensive matrix cracking occurs at the first cracking strength of the composite $f_{t}$. The slope of the pre-peak straight portion of the curve is approximated 


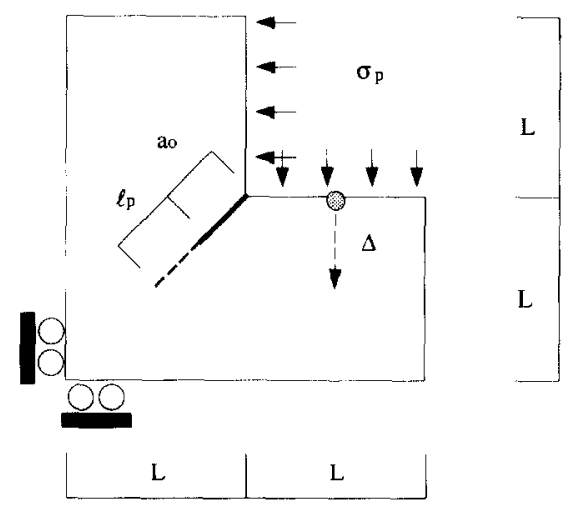

Fig. 5. Geometric and loading configuration of analytical shear key model.

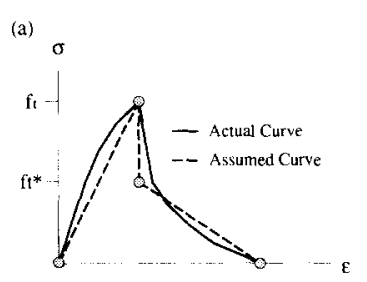

(b)

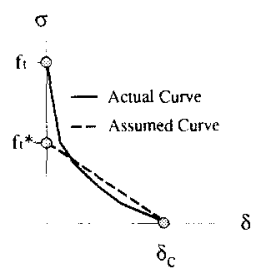

Fig. 6. Softening Model $1\left(f_{i} / f_{t}^{*}=0.5\right)$ : (a) Composite uniaxial curve and (b) Stress-separation curve.

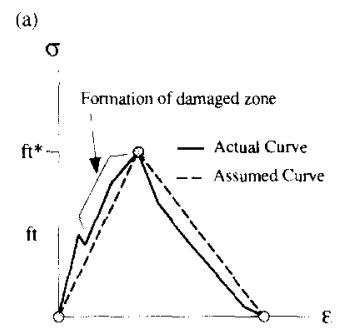

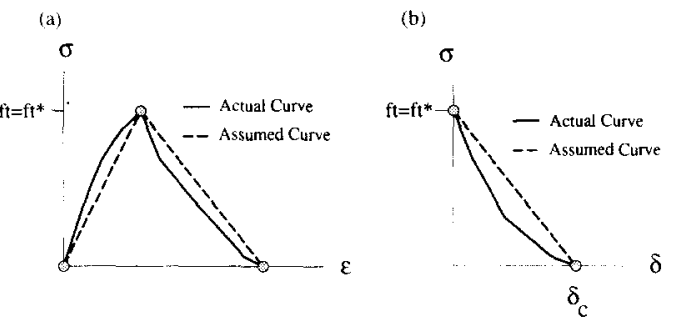

Fig. 7. Softening Model $2\left(f_{i} / f_{i}^{*}=1.0\right)$ : (a) Composite uniaxial curve and (b) Stress-separation curve.

(b)

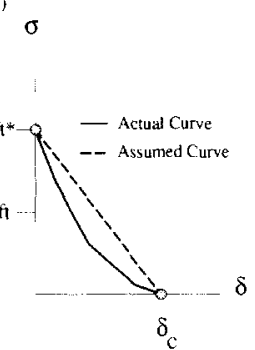

Fig. 8. Softening Model $3\left(f_{1} f_{1}^{*}=2.0\right)$ : (a) Composite uniaxial curve and (b) Stress-separation curve.
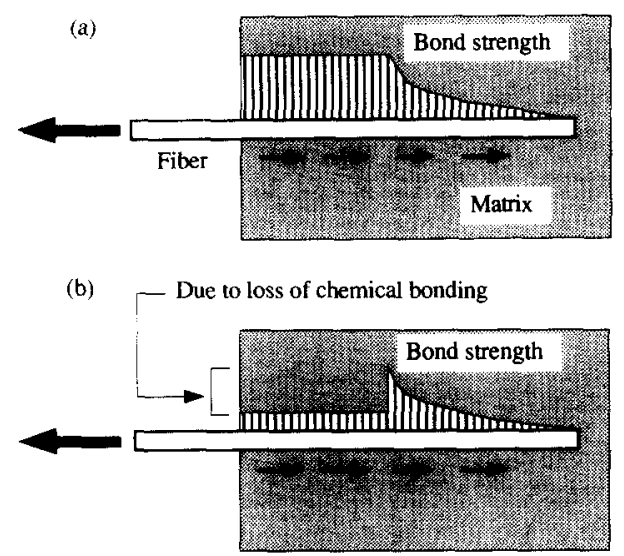

Fig. 9. Bond stress distribution in the interface between a fiber and a matrix dominated by (a) Frictional bonding and (b) Frictional bonding plus chemical bonding. 
by the rule of mixtures based on matrix and fiber moduli. After $f_{t}$ is reached, the stress drops unstably to the post cracking strength of the composite $f_{t}^{*}$. After $f_{i}^{*}$ is reached, fibers tend to be pulled out gradually showing softening behavior. In this case, after matrix cracking fibers cannot support all the load (e.g. the fiber volume fraction is too small to carry the load shed by the matrix), thus leading to the sudden load drop. Alternatively, after matrix cracking fibers can support a slightly higher load before there is a sudden drop in stress due to unstable fiber debonding in a case where there is both chemical bond and friction at the interface.

In the softening model 2, extensive matrix cracking occurs at the first cracking strength of the composite $f_{t}$. The slope of the pre-peak straight portion of the curve is approximated by the rule of mixtures based on matrix and fiber moduli as well. After $f_{t}\left(=f_{t}^{*}\right)$ is reached, fibers tend to be pulled out gradually showing softening behavior. In this case, after matrix cracking fibers can support a slightly higher load before the pull-out occurs. The interface is purely frictional so there is no sudden load drop.

In the softening model 3 , the slope of the initial straight portion of the curve is closely approximated by the rule of mixtures based on matrix and fiber moduli as well. Extensive matrix cracking, often involving a small stress drop [8], occurs at the first cracking strength of the composite $f_{t}$. Subsequently, the matrix becomes permeated by many equally spaced cracks that traverse the full cross-section of the specimens. This portion could be called a damage zone. Under continued loading, the fibers alone provide most of the subsequent stiffness. The post cracking strength of the composite $f_{t}^{*}$ would be associated with the maximum stress carried by the fiber/matrix interface. Thus, fibers can support a much higher load before the pull-out occurs. Again, in this case a purely friction interface is assumed. In model 3, multiple cracking of the matrix may occur beyond the first cracking strength. Due to this multiple cracking phenomenon, fracture analysis of a single crack is not strictly valid. Thus, the correct structural behavior of model 3 could be obtained by an approach which can take into consideration the multiple cracking phenomenon. At the present stage, for simplicity, we assume a line crack model which may not be valid as the value of $f_{t}^{*} / f_{t}$ increases to 2.0. Therefore, the numerical results obtained in the region of $f_{t}^{*} / f_{t}=1.0-2.0$ could be correct only when the value of $f_{t}^{*} / f_{t}$ is close to 1.0 . The interpretation of the results in the region of $f_{t}^{*} / f_{t}=1.0-2.0$ are based on this restricted assumption. As the value of $f_{t}^{*} / f_{t}$ increases from 1.0 to 2.0 , the results become more conservative.

A material characteristic length $l_{\text {ch }}$ proposed by Hillerborg [9] is used to normalize all length dimensions. Although the material characteristic length has no physical meaning, it can be advantageously used to reduce structural lengths to dimensionless forms. In addition, all stress dimensions are normalized by a first cracking strength $f_{t}$. Some examples are described as follows.

Critical crack tip opening displacement (CTOD)

$$
\left(\frac{\delta_{c}}{l_{c h}}\right)=\frac{2}{\left(\frac{E}{f_{t}}\right)\left(\frac{f_{t}^{*}}{f_{t}}\right)}
$$

Fracture energy

$$
\frac{G_{f}}{f_{t} l_{c h}}=\frac{1}{2}\left(\frac{f_{t}^{*}}{f_{t}}\right) \frac{2}{\left(\frac{E}{f_{t}}\right)\left(\frac{f_{t}^{*}}{f_{t}}\right)}=\frac{1}{\left(\frac{E}{f_{t}}\right)}
$$




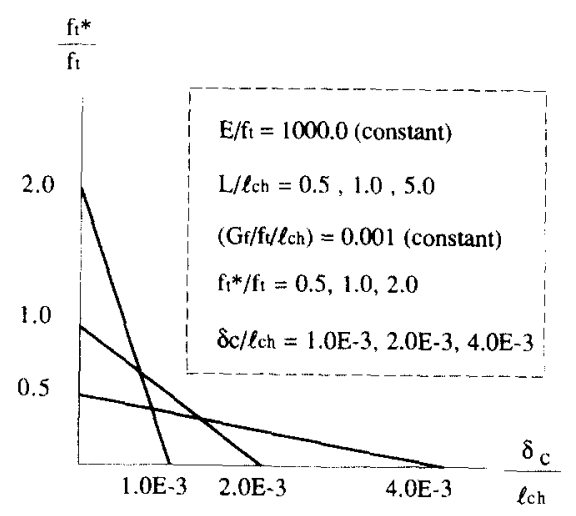

Fig. 10. Linear model of stress-separation curves.

Fracture toughness

$$
\frac{K_{\mathrm{IC}}}{f_{t} \sqrt{l_{c h}}}=\frac{1}{\sqrt{1-v^{2}}} \approx 1.0 .
$$

In this analysis, the residual strength of the structure is defined by the applied load $\sigma_{p} / f_{t}$ where the process zone is fully developed or when the crack opening displacement at the physical crack tip is equal to the assumed critical crack tip opening displacement in the stress-separation curve. Specifically, the residual strength could be calculated by first specifying the crack opening displacement at the physical crack tip to be the critical opening displacement. Then, the process zone length is assumed and numerical iteration is carried out until there is no singularity at the fictitious crack tip of the process zone. The process zone at this stage is fully developed.

The variations of material properties in the analysis are shown in Fig. $10 . \mathrm{L} / \mathrm{I}_{\mathrm{ch}}$ is a geometric size and $f_{t}^{*} / f_{t}$ is a parameter of the stress-separation curves and $E / f_{t}$ is a material parameter of the composite as well as of the stress-separation curve. This figure can be understood as follows.

Let $L / l_{c h}$ and $E / f_{t}$ (in the analysis, $E / f_{t}$ is fixed 1000.0) be fixed. In changing $f_{t}^{*} / f_{t}$ from 0.5 to 2.0 , the composite maintains the fracture energy $\left(G_{f} / f_{t}\right) / l_{\text {ch }}$ constant (since $\left(G_{f} / f_{t}\right) / l_{\text {ch }}$ is inversely proportional to $E / f_{t}$, and also maintain the fracture toughness $\left(K_{\mathrm{IC}} / f_{t}\right) /\left(l_{c h}\right)^{0.5}$ constant (since the present normalization always makes it constant).

All the following analysis results and interpretations are based on this restricted variation.

\section{Residual strength diagram}

Residual strength could be defined as the remaining strength of structures under the presence of cracks $[10]$. Thus, the residual strength diagram could show the maximum load carrying capacity of a cracked structure. This value is the same as the peak load and is different from the traction free crack propagation load (see Fig. 3). Thus, the residual strength is not directly related to the propagation of traction free crack. However, the difference btween the peak load and the traction free crack propagation load may be fairly small in the linear stress-separation model which was discussed in the previous section (see also Fig. 3). Therefore, we define the residual strength for each traction free crack length $a_{0}$ to be the traction free crack propagation 
load with fully developed process zone in this analysis. The loads in the obtained load-deflection curves are defined the same as well.

Obtained residual strength diagrams are shown in Figs. 11-13. The LEFM results show the behavior of composites when they are calculated by LEFM concept. Therefore, the difference between LEFM results and NLEFM results indicate the conditions for validity of LEFM in the numerical analysis.

From the analysis, the following results are observed (Here, it should be noted that the validity of results in the region of $f_{t}^{*} / f_{t}=1.0-2.0$ decreases as the value of $f_{t}^{*} / f_{t}$ increases since the damage zone size could increase as the value of $f_{t}^{*} / f_{t}$ increases).

(1) In the region of a small initial crack length $a_{0}\left(a_{0} / l_{c h}<0.1\right)$, the behavior of composites is insensitive to the presence of a crack.

(2) The residual strength $\sigma_{p} / f_{t}$ for a given crack length increases as the value of $f_{t}^{*} / f_{t}$ increases (or the value of $\delta_{c} / l_{c h}$ decreases).

(3) The NLEFM results are closer to LEFM results as the slope of the linear line in the stress-separation curve becomes more negative (the value of $\delta_{c} / l_{c h}$ decreases or $f_{t}^{*} / f_{t}$ increases in Fig. 10).

(4) The above behavior of composites is the same even if $L / l_{c h}$ is changed. However, as the value of $L / l_{c h}$ increases, the NLEFM results become closer to LEFM results. This is because something like a small scale yielding (SSY) condition can be approached faster with a larger structure.

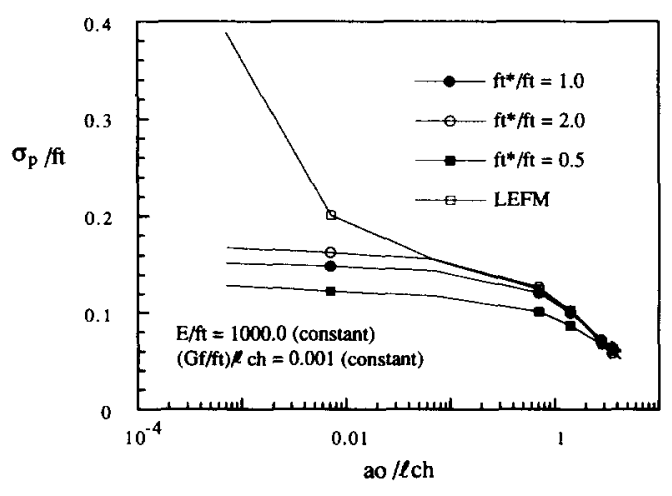

Fig. 11. Residual strength diagram $\left(L / l_{\text {ch }}=5.0\right)$.

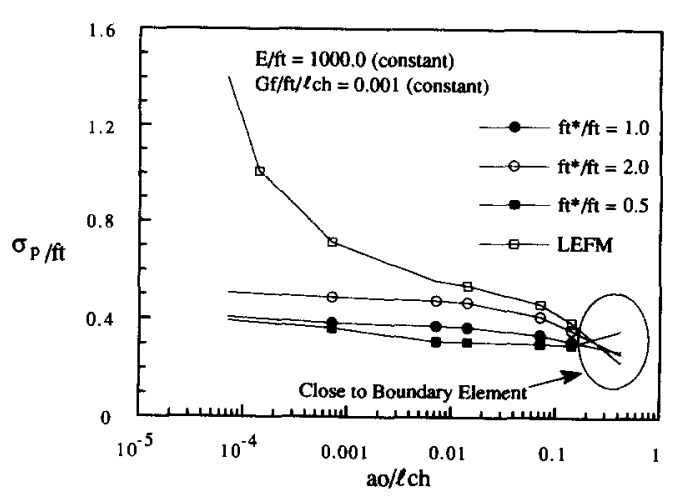

Fig. 13. Residual strength diagram $\left(L / l_{c h}=0.5\right)$.

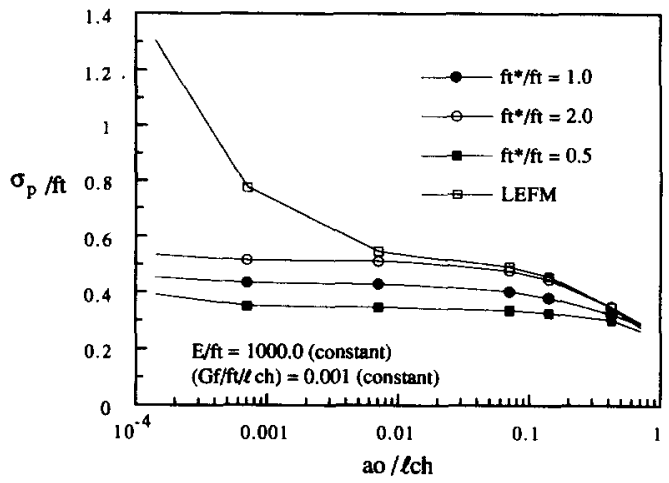

Fig. 12. Residual strength diagram $\left(L / l_{c h}=1.0\right)$.

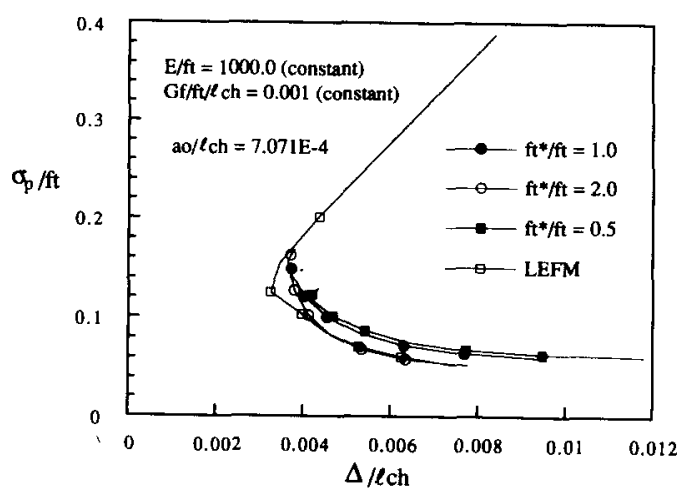

Fig. 14. Load deflection curve after the peak load $\left(L / l_{c h}=5.0\right)$. 
(5) Valid condition for LEFM approach is observed for relatively large crack length $\left(a_{0} / l_{c h}>0.01\right)$. This is because as $a_{0} / l_{c h}$ gets larger, the ratio of the process zone size to the initial crack length becomes smaller.

\section{Load-deflection curve}

Obtained load-deflection curves associated with steady-state crack propagation are shown in Figs. 14-16. The values of loads in the figures are identical to those in the residual strength diagrams shown in Figs. 11-13. The pre-peak behavior is neglected in the figures. The maximum load shown could be slightly lower than the actual maximum load (=the peak load in Fig. 3) since the maximum load on the graph is obtained from the traction free crack propagation load as discussed previously. However, one can see the stability of shear key structures with a softening process zone under effectively bending loads after the peak load. From the analysis, the following results are obtained (Here, it should be noted again that the validity of results in the region of $f_{t}^{*} / f_{t}=1.0-2.0$ decreases as the value of $f_{t}^{*} / f_{t}$ increases).

(1) Brittle materials usually show a snap back phenomena during crack propagation. Such a behavior is shown by the curves for LEFM in Figs. 14-16. For composites (with behavior shown in the same figures), there is no significant snap back and their overall behavior therefore seems to be stable.

(2) The structures with lower value of $\delta_{c} / l_{c h}$ or higher value of $f_{t}^{*} / f_{t}$ (the more negative the slope of the stress-separation curve in the present case) have a sudden drop of load carrying capacity after the snap back action resulting in relatively unstable behavior.

(3) The structures with higher value of $\delta_{c} / l_{c h}$ or lower value of $f_{t}^{*} / f_{t}$ have larger deformation for the same physical crack length.

(4) The NLEFM results with lower value of $\delta_{c} / l_{c h}$ or higher value of $f_{t}^{*} / f_{t}$ are closer to LEFM results. This behavior could also be observed in the residual strength diagram.

(5) The structure with higher value of $\delta_{c} / l_{c h}$ or lower value of $f_{t}^{*} / f_{t}$ (more stable structures in the present case) shows the lower maximum load in the load-deflection curve and shows larger deflection at the maximum load. This can be explained from the work of Li and Liang [3] that shows the initial stiffness of composites are the same and more ductile structures tend to

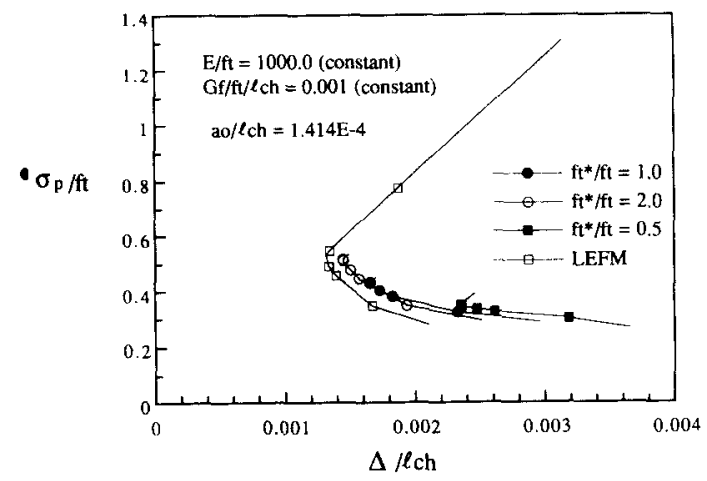

Fig. 15. Load deflection curve after the peak load $\left(L / l_{c h}=1.0\right)$.

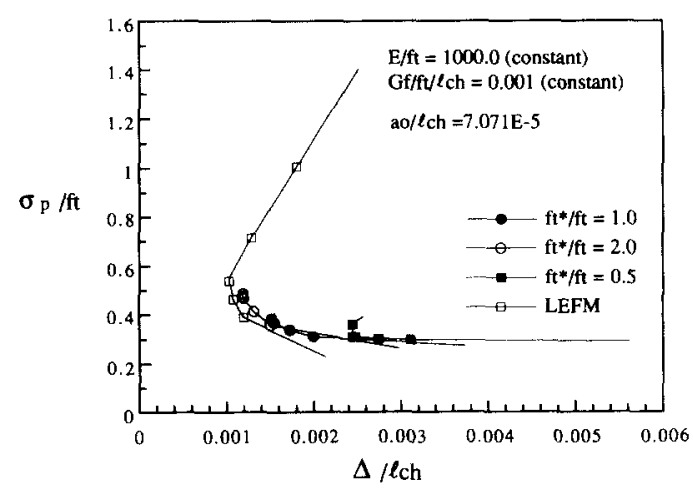

Fig. 16. Load deflection curve after the peak load $\left(L / l_{c h}=0.5\right)$. 
reduce the stiffness earlier and the difference between displacement at the peak load and that at the traction free crack propagation load increases as ductility increases (see Fig. 3).

(6) The above behavior of composites is the same even if $L / l_{c h}$ is changed. However, as the value of $L / l_{c h}$ increases, the NLEFM results become closer to LEFM results as well as the residual strength diagram. This is because something like a small scale yielding (SSY) condition can be approached faster with a larger structure.

\section{Behavior of process zone size}

The steady-state process zone size $\left(l_{p}\right)_{s s}$ may be estimated by assuming the stress intensity factor induced by the applied force equal to the fracture toughness of the composite in the equilibrium relation of stress intensity (Barenblatt's approach) and assuming that the fiber bridging stresses vary linearly from $f_{t}$ to 0 in the process zone. This procedure was first used by Palmer and Rice [11] who studied the 'slip-weakening' process in consolidated clay slopes under shear deformation. They obtained

$$
\left(l_{p}\right)_{s s}=\frac{9 \pi}{32}\left(\frac{E}{1-v^{2}}\right)\left(\frac{G_{f}}{f_{t}^{2}}\right) .
$$

Evaluation of the above equation gives $\left(l_{p}\right)_{s s} / l_{c h}=0.9$. This relation is extremely simple. However, this equation may not precisely describe the present NLEFM results since they assumed a linear stress distribution in the process zone. In addition, they did not consider geometric size in their analysis which might also affect their results.

The process zone size $l_{p} / l_{c h}$ versus crack length $a_{0} / l_{c h}$ curves obtained from the NLEFM analysis are shown in Figs. 17-19 for the case of $E / f_{t}=1000.0$. From the analysis, the following results are observed (Here, it should be noted again that the validity of results in the region of $f_{t}^{*} / f_{t}=1.0-2.0$ decreases as the value of $f_{i}^{*} / f_{t}$ increases).

(1) Process zone size is very insensitive to the crack size in the small crack region $\left(a_{0} / l_{c h}<0.1\right)$. This phenomenon is especially evident in the case of the lower value of $\delta_{c} / l_{c h}$ or higher value of $f_{t}^{*} / f_{t}$.

(2) The case with lower value of $\delta_{c} / l_{c h}$ or higher value of $f_{t}^{*} / f_{t}$ (more negative slope of line in the stress-separation curve in the present case) has the lower process zone size.

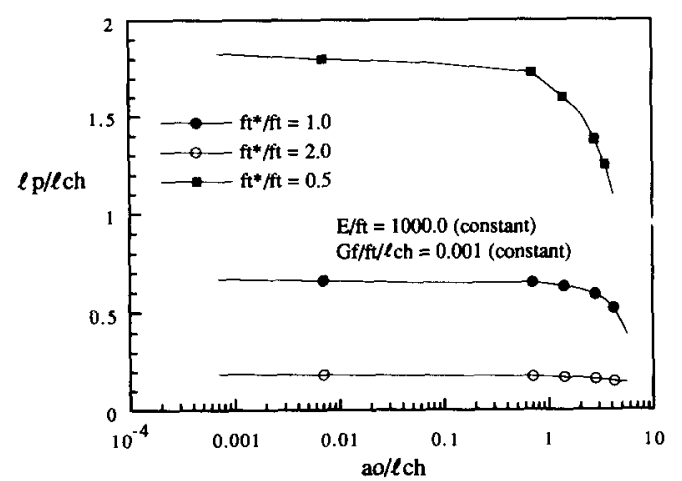

Fig. 17. Process zone size $\left(L / I_{c h}=5.0\right)$.

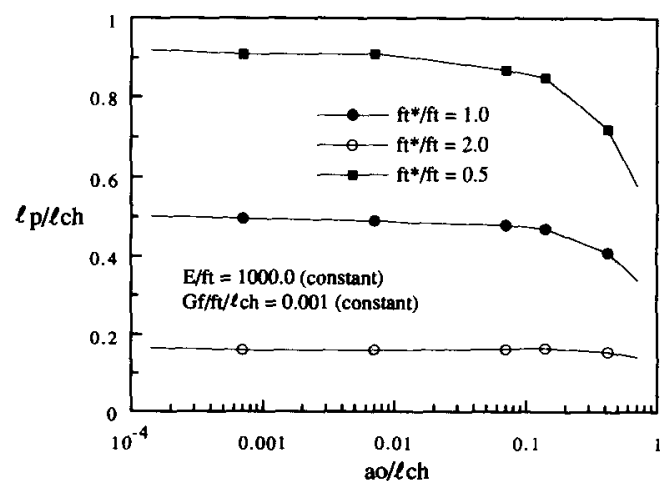

Fig. 18. Process zone size $\left(L / l_{c h}=1.0\right)$. 


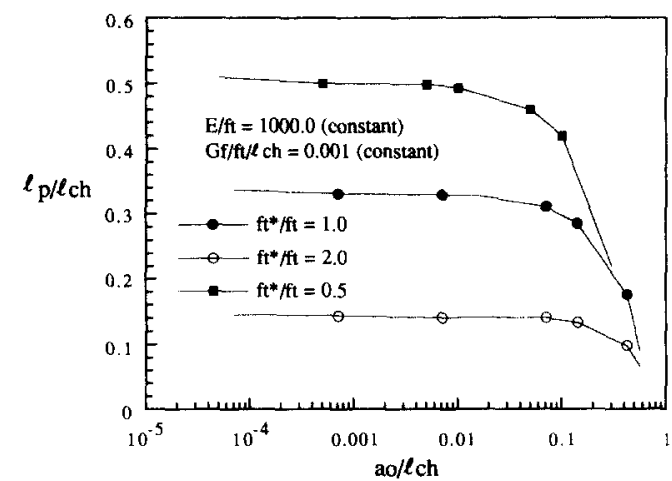

Fig. 19. Process zone size $\left(L / l_{c h}=0.5\right)$.

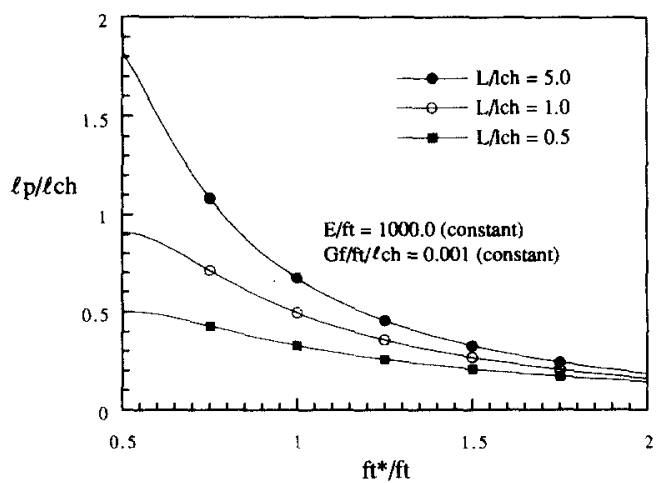

Fig. 20. Normalized process zone size for different value of $L / l_{c h}$.

(3) Process zone size tends to drop suddenly at the large crack region. This phenomenon is especially evident in the case of the higher value of $\delta_{c} / l_{c h}$ or lower value of $f_{t}^{*} / f_{t}$.

(4) The above behavior of process zone size is the same even if the value of $L / l_{c h}$ is changed. However, as $L / l_{c h}$ increases, the process zone size increases.

Overall, the obtained results are different from Palmer and Rice's simple relation. This is because we assumed the linear stress-separation curves resulting in nonlinear stress distribution in the process zone, while they assumed linear stress distribution in the process zone.

From the NLEFM results, the process zone size $l_{p} / l_{c h}$ could be calculated as a function of $f_{t}^{*} / f_{t}$ and $L / l_{c h}$. The objective here is to find numerically a simple relation among the process zone size, the stress-separation curve and geometric size assuming a linear stress-separation curve and fixed loading configuration. We try to find a simple approximate relation between $l_{p} / l_{c h}, f_{t}^{*} / f_{t}$ and $L / l_{c h}$ with cohesive zone approach. Unknown constants will be present in such a simple relation. By plotting the numerical results obtained from the NLEFM analysis, a regression analysis could be carried out to obtain the unknown constants.

Cohesive zone approach indicates the stress intensity factors induced by external loading must be cancelled out by the negative stress intensity factors induced by the cohesive stresses along the process zone. Actually, this theory cannot be valid for certain composites where a singularity is maintained by the relatively high toughness matrix material. In the present analysis, this effect is neglected to obtain the simple relation between $l_{p} / l_{c h}, f_{t}^{*} / f_{t}$ and $L / l_{\text {ch }}$.

Using the Dugdale model [12] which assumes a constant bridging stress distribution at the process zone, one could obtain the following simple relations by mathematical approximation (see Appendix I).

$$
\frac{1.0}{\frac{l_{p}}{l_{c h}}+1.0} \propto 1.0-\frac{1}{2}\left(C 2 \beta+\frac{C 1 \beta}{\frac{f_{t}^{*}}{f_{t}}}\right)^{2},
$$

where $\beta$ could be related to $1 /\left(L / l_{c h}\right)$, and $C 1$ and $C 2$ are constant. The reason for the use of the Dugdale model assuming uniform bridging stress is that the purpose of the simple analysis is to identify parameters governing the size of the process zone. The actual dependence of process zone size on such parameters will be obtained through empirical fitting to results from numerical computation. 


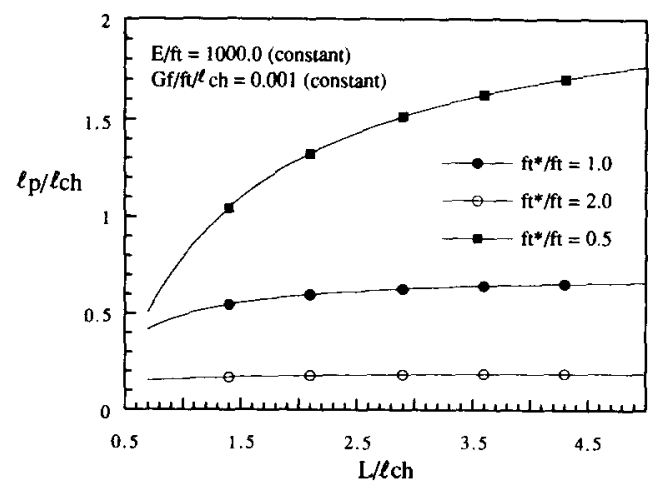

Fig. 21. Normalized process zone size for different value of $f_{t} / f_{t}^{*}$.

Using this relation, one can carry out a regression analysis with the NLEFM numerical results. Then, one could obtain the behavior of $l_{p} / l_{c h}$ in terms of $f_{t}^{*} / f_{t}$ and $L / l_{c h}$ shown in Fig. 20 and Fig. 21. As discussed previously, the validity of these figures in the region of $f_{t}^{*} / f_{t}=1.0-2.0$ could decrease as the value of $f_{t}^{*} / f_{t}$ increases.

Actually, these quantitative relations should be assessed with experimental verification, and are valid for only the present cases. However, these results might be used for several types of shear key structures with a softening process zone like short fiber reinforced ceramic structures, especially in the small crack region with large size structures if the behavior of the structures is dominated by mode $I$.

\section{Conclusion}

In this paper, we investigated the fracture behavior of short fiber reinforced ceramic shear key structures by means of the fracture mechanics approach. Structural stability in relation to crack growth of shear key structures under effective bending loads was studied through a residual strength diagram and a load deflection curve. In addition, the behavior of process zone size in the structure was investigated, and quantified numerically.

From this analytical study, the following conclusions could be drawn.

(1) The shear key structures with a softening process zone behave stably under the presence of relatively small crack. Specifically, the residual strength of cracked shear key structures is insensitive to the relatively small crack, and no significant snap back action is observed in the load-deflection curves. These phenomena are not influenced by the structural size.

(2) The residual strength of shear key structures with a softening process zone can be predicted by LEFM calculation when the value of $a_{0} / l_{c h}$ is larger than approximately 0.01 .

(3) The process zone size of shear key structures with a softening process zone is quantitatively predicted by a relatively simple numerical manner. The derived quantitative equation of process zone size is expressed in terms of the stress-separation curve and the structural size of shear key structures. These analytical results might make further numerical work on the behavior of fiber reinforced ceramic structures under tensile loading easier. However, since these quantities are obtained numerically and the calculation is achieved by a simple model like the Dugdale model, these results should be compared with the experimental works. 
(4) Present analytical studies indicated short fiber reinforcement for ceramics with adequate toughness and a large critical separation $\delta^{*}$ could eliminate a catastrophic failure or unstable fracture behavior of ceramic structures, and also indicated potentially the plausible applicability of ceramics as construction materials.

\section{Appendix I}

\section{Calculation of process zone size}

The stress intensity factors induced by external loading (see Fig. 5)

$$
K_{1}=\beta_{1} \sigma_{p} \sqrt{\pi\left(a_{0}+l_{p}\right)}
$$

where $\beta_{1}$ is a geometric factor and can be related to $1 / L$.

The stress intensity factors induced by the cohesive stresses assumed to distribute uniformly with the magnitude of $f_{t}^{*}$ along the process zone

$$
K_{2}=\beta_{2} 2 f_{t}^{*} \sqrt{\frac{a_{0}+l_{p}}{\pi}} \cos ^{-1}\left(\frac{a_{0}}{a_{0}+l_{p}}\right) \text {, }
$$

where $\beta_{2}$ is a geometric factor and could be related to $1 / L$.

Equating $K_{1}$ to $K_{2}$

$$
\beta_{1} \sigma_{p} \sqrt{\pi\left(a_{0}+l_{p}\right)}=\beta_{2} 2 f_{t}^{*} \sqrt{\frac{a_{0}+l_{p}}{\pi}} \cos ^{-1}\left(\frac{a_{0}}{a_{0}+l_{p}}\right)
$$

namely

$$
\cos \left(\frac{\beta_{1}}{\beta_{2}} \frac{\sigma_{p}}{2 f_{i}^{*}} \pi\right)=\frac{a_{0}}{a_{0}+l_{p}},
$$

then, normalizing the variables by $l_{c h}$ and $f_{t}$

$$
\frac{\frac{l_{p}}{l_{c h}}}{\frac{a_{0}}{l_{c h}}}=\sec \left(\frac{\frac{\sigma_{p}}{2} \pi \beta \frac{\frac{f_{t}}{f_{t}^{*}}}{f_{t}}}{2}\right)-1
$$

where $\beta$ equals $\beta_{1} / \beta_{2}$ and can be related to $1 /\left(L / l_{c h}\right)$.

Fixing $a_{0} / l_{c h}$ in the region of small crack

$$
\frac{l_{p}}{l_{c h}}+1.0 \propto \sec \left(\frac{1}{2} \pi \beta \frac{\frac{\sigma_{p}}{f_{t}}}{\frac{f_{t}^{*}}{f_{t}}}\right)
$$


Assuming $\sigma_{p} / f_{t}$ is linearly proportional to $f_{t}^{*} / f_{t}$ (This is slightly different from the NLEFM results)

$$
\frac{l_{p}}{l_{c h}}+1.0 \propto \sec \left(\left(C 1+C_{2} \frac{f_{t}^{*}}{f_{t}}\right) \frac{\beta}{\frac{f_{t}^{*}}{f_{t}}}\right)=\sec \left(C 2 \beta+\frac{C 1 \beta}{\frac{f_{t}^{*}}{f_{t}}}\right) .
$$

Neglecting the higher order terms in the series, one can obtain

$$
\frac{1.0}{\frac{l_{p}}{l_{c h}}+1.0} \propto 1.0-\frac{1}{2}\left(C 2 \beta+\frac{C 1 \beta}{\frac{f_{t}^{*}}{f_{t}}}\right)^{2}
$$

where $C 1$ and $C 2$ are constant.

The relation between the process zone size and stress-separation curve with fixed $L / l_{c h}$ and $E / f_{t}$ is obtained as follows.

Fixing $\beta$, one can obtain

$$
\frac{1.0}{\frac{l_{p}}{l_{c h}}+1.0}=f_{1}\left\{\frac{1}{\frac{f_{t}^{*}}{f_{t}}},\left(\frac{1}{\frac{f_{t}^{*}}{f_{t}}}\right)^{2}\right\}
$$

Using this relation, one can carry out a regression analysis with the NLEFM numerical results. The obtained results are as follows

$$
\begin{aligned}
& L / l_{c h}=5.0: \frac{1.0}{\frac{l_{p}}{l_{c h}}+1.0}=1.1721-0.73915\left(\frac{1}{\frac{f_{t}^{*}}{f_{t}}}\right)+0.165\left(\frac{1}{\frac{f_{t}^{*}}{f_{t}}}\right)^{2} ; \\
& L / l_{c h}=1.0: \frac{1.0}{\frac{l_{p}}{l_{c h}}+1.0}=1.1365-0.62959\left(\frac{1}{\frac{f_{t}^{*}}{f_{t}}}\right)+0.16157\left(\frac{1}{\frac{f_{t}^{*}}{f_{t}}}\right)^{2} \\
& L / l_{c h}=0.5: \frac{1.0}{\frac{l_{p}}{l_{c h}}+1.0}=1.0515-0.40683\left(\frac{1}{\frac{f_{t}^{*}}{f_{t}}}\right)+0.10721\left(\frac{1}{\frac{f_{t}^{*}}{f_{t}}}\right)^{2}
\end{aligned}
$$

The relation between the process zone length and geometric size with fixed $f_{t}^{*} / f_{t}$ and $E / f_{t}$ are obtained as follows. 
Fixing $f_{t}^{*} / f_{\mathrm{t}}$ in (13), one can obtain

$$
\frac{1.0}{\frac{l_{p}}{l_{c h}}+1.0}=f_{2}\left\{\beta, \beta^{2}\right\}
$$

where $\beta$ can be related to $1 /\left(L / l_{\text {ch }}\right)$.

Using this relation, one can carry out a regression analysis with the NLEFM numerical results. The obtained results are as follows

$$
\begin{aligned}
& f_{t}^{*} / f_{t}=1.0: \frac{1.0}{\frac{l_{p}}{l_{c h}}+1.0}=0.58326+0.091684\left(\frac{1}{\frac{L}{l_{c h}}}\right)-0.0037891\left(\frac{1}{\frac{L}{l_{c h}}}\right)^{2} \\
& f_{t}^{*} / f_{t}=2.0: \frac{1.0}{\frac{l_{p}}{l_{c h}}+1.0}=0.83251+0.037733\left(\frac{1}{\frac{L}{l_{c h}}}\right)-0.0082662\left(\frac{1}{\frac{L}{l_{c h}}}\right)^{2} \\
& f_{t}^{*} / f_{t}=0.5: \frac{1.0}{\frac{l_{p}}{l_{c h}}+1.0}=0.31128+0.2520\left(\frac{1}{L}\right)-0.037263\left(\frac{1}{\frac{L}{L}}\right)^{2}
\end{aligned}
$$

\section{References}

1. Y. Kaneko and V.C. Li, in Serviceability and Durability of Construction Materials, Proceedings of the First Engineering Congress, Denver, B.A. Suprenant (ed.) 1 (1990) 795-803.

2. Y. Kaneko and V.C. Li, Construction \& Building Materials 6, No. 1 (1992) 2-7.

3. V.C. Li and E. Liang, Journal of Engineering Mechanics 112, No. 6 (1986) June.

4. G.I. Barenblatt, in Advances in Applied Mechanics, H.L. Dryden and T. von Karman (eds.) 7 (1962).

5. H.C. Chan, V.C. Li and H.H. Einstein, International Journal of Fracture 45 (1990) 263-282.

6. O.M.L. Reyes, Numerical Modelling of Fracture Propagation in Tension Softening Materials, S.M. thesis, Massachusetts Institute of Technology (1987).

7. P. Bartos, Journal of Materials Science 15 (1980) 3122-3128.

8. B. Budiansky, J.W. Hutchinson and A.G. Evans, Journal of the Mechanics and Physics of Solids 34, No. 2 (1986) $167-189$

9. A. Hillerborg, in Fracture Mechanics of Concrete, F.H. Whittman (ed.), Elsevier Science Publishers, The Netherlands (1934) 223-249.

10. D. Broek, The Practical Use of Fracture Mechanics, Kluwer Academic Publishers, Dordrecht (1988).

11. A.C. Palmer and J.R. Rice, Proceedings of the Royal Society of London, A332 (1973) 527.

12. D.S. Dugdale, Journal of the Mechanics and Physics of Solids 8 (1960) 100-108. 